\title{
Tendencias y tipos de aprendizaje en MALL: una revisión sistemática de la literatura (2012-2016) \\ Trends and types of learning in MALL: a systematic literature review (2012- 2016)
}

\author{
Andrea Calderón Márquez ${ }^{1}$, Manuel Palomo Duarte ${ }^{2}$, Anke Berns ${ }^{1}$, Juan Manuel Dodero ${ }^{2}$ \\ andrea.calderonmarquez@alum.uca.es, manuel.palomo@uca.es, anke.berns@uca.es, juanma.dodero@uca.es \\ ${ }^{1}$ Departamento de Filología Francesa e Inglesa \\ Universidad de Cádiz \\ Cádiz, España \\ ${ }^{2}$ Departamento de Ingeniería Informática \\ Universidad de Cádiz \\ Cádiz, España
}

\begin{abstract}
Resumen-La creciente expansión de las Tecnologías de la Información y la Comunicación (TIC) no solo ha creado nuevos modelos de comunicación e interacción, sino también de enseñanzaaprendizaje. En la enseñanza de idiomas esto se ha plasmado en el uso de ordenadores como soporte para el aprendizaje (CALL) y, más recientemente, en el uso de dispositivos móviles (MALL). A pesar de que las primeras aplicaciones de MALL aparecieran hace más de dos décadas, sigue siendo un campo poco explorado, teniendo en cuenta las enormes posibilidades de interacción e innovación que ofrecen hoy día dispositivos móviles como los smartphones. Con el objetivo de identificar, por un lado, tendencias actuales y, por otro, abrir posibles futuras líneas de investigación, este artículo presenta una revisión sistemática de la literatura (RSL), a partir de trabajos de investigación publicados en revistas, congresos y libros especializados, entre 2012 y 2016. De especial interés es la identificación de los tipos de aprendizaje, que promueven las apps que se encuentran actualmente en la literatura especializada. El trabajo pretende dar continuidad a otras RSL previas, para así obtener una perspectiva del actual estado del arte de MALL, además de identificar los desafíos y retos a los que se sigue enfrentando.
\end{abstract}

Palabras clave:aprendizaje de lenguas mediante tecnología móvil, smartphones, apps, Revisión Sistemática de la Literatura (RSL).

Abstract-The growing expansion of Information and Communication Technology (ICT) has not only given rise to new ways of communicating and interacting, but also of teaching and learning. In foreign language teaching this has led to the rise of new disciplines such as Computer Assisted Language Learning (CALL) and, more recently, Mobile Assisted Language Learning (MALL). Although the first MALL implementations appeared more than two decades ago, it is still a barely explored area, bearing in mind the possibilities offered by mobile devices such as smartphones. As the purpose of this study is, on the one hand, to identify current trends and, on the other hand, to contribute to future research, this article presents a systematic literature review (SLR), based on research studies published in journals, conferences and specialised books between 2012 and 2016. Special attention is given to the type of learning supported by the apps that can be found within the specialised literature. This study aims to add to previous SLR in order to gain insight into the state of the art of MALL, as well as to identify the challenges it still faces.

Keywords: MALL, smartphones, apps, Systematic Literature Review (SLR).

\section{INTRODUCCIÓN}

El punto de partida de este estudio es la creciente expansión de las tecnologías móviles y, en concreto, de los smartphones y tabletas para mejorar los procesos de enseñanza-aprendizaje de idiomas (Briz-Ponce et al., 2016; Ramírez-Montoya y García-Peñalvo, 2017). Su popularidad se debe indudablemente a que ofrecen nuevas posibilidades para facilitar un aprendizaje más flexible y ubicuo. Y no solo eso, sino que también permiten crear nuevos entornos de aprendizaje, basados en la interacción y colaboración entre varios usuarios, a partir de numerosas herramientas multimedia (Kukulska-Hulme, 2005; Kukulska-Hulme y Shield, 2008; Pinto-Llorente et al., 2016).

A fin de identificar, por un lado, actuales tendencias de uso y, por otro, esbozar futuras líneas de investigación, el presente estudio propone hacer una revisión sistemática de la literatura (RSL) a partir de diferentes estudios de investigación, publicados entre enero del 2012 y diciembre del 2016. Para el análisis, se utilizaron fuentes como revistas especializadas, actas de congreso y libros relacionados con la temática del aprendizaje de idiomas a través de dispositivos móviles (MALL). En el caso de MALL, tratándose de un campo en continua expansión y con numerosos retos por delante, el presente estudio pretende dar continuidad a las RSL realizadas por Chinnery (2006), Viberg y Grönlund (2012), Burston (2013, 2014 \& 2015) y Duman (2015), a fin de obtener una perspectiva del actual estado del arte de MALL, identificando las tendencias más recientes junto a futuras líneas de investigación.

El interés en el uso de dispositivos móviles para apoyar los procesos de enseñanza-aprendizaje ha ido creciendo paulatinamente desde sus inicios en los años 90 hasta hoy día. Prueba de ello no solo es el creciente número de apps, que encontramos en plataformas como Google Play Store (Heil et al., 2016), sino también el creciente número de investigaciones en el ámbito de MALL. De esta manera, las publicaciones relacionadas con MALL parecen aumentar y madurar especialmente en los últimos años, sumando en el año 2012 más de 570 publicaciones desde sus inicios en 1994 (Burston, 2013 \& 2014). En cuanto a RSL, los trabajos más notables son 
los publicados por Chinnery (2006), Viberg y Grönlund (2012), Burston (2013, 2014 \& 2015) y Duman et al. (2015). En "Going to the MALL: Mobile Assisted Language Learning” (2006), Chinnery analiza las publicaciones sobre el aprendizaje de idiomas mediante dispositivos móviles, identificando, entre otros aspectos, los beneficios y retos a los que se enfrenta su puesta en práctica (p. ej. el tamaño limitado de las pantallas, la duración de la batería, la limitada calidad audiovisual). Al estudio de Chinnery le sigue en el 2012 el estudio de Viberg y Grönlund, donde los autores hacen una revisión sistemática de la literatura publicada sobre MALL entre los años 2007 y 2012. En ella, señalan que la mayor parte de los estudios se centran en vocabulario, así como en comprensión y expresión oral, destacando la utilidad de los dispositivos móviles a la hora de, por ejemplo, dotar al alumno con la posibilidad de formar parte de la creación de nuevo contenido. Tras esta, nos encontramos, en 2013, con la bibliografía anotada de Burston, en la que el autor lista y resume un total de 350 estudios sobre implementaciones MALL, publicados entre 1994 y 2012. Asimismo, Burston examina en su trabajo, "Twenty years of MALL project implementation: A meta-analysis of learning outcomes" (2015), los resultados de dichas implementaciones MALL, proponiendo una serie de criterios para validar un determinado proyecto. Algunos de los criterios son: la duración del experimento, el número de participantes y la existencia de fallos en el diseño (p.ej. errores de monitorización, la presencia de variables fuera de control, etc.). En cuanto a aspectos meramente pedagógicos, destaca el estudio "MALL: the pedagogical challenges" que fue realizado por el mismo autor (Burston, 2014) y que analiza las tendencias y retos pedagógicos de MALL. Los resultados del estudio indican que, pese a ser una disciplina que goza de creciente interés, sigue sin explorar muchas de las posibilidades que hoy día ofrece la mayoría de los dispositivos móviles para ir complementando y enriqueciendo los tradicionales procesos de enseñanza-aprendizaje. Al trabajo de Burston le sigue, finalmente, otro estudio realizado por Duman et al., (2015), "Research trends in mobile assisted language learning from 2000 to 2012”, en el que los autores identifican las tendencias más destacables de las investigaciones publicadas entre los años 2000 y 2012. Entre estas, los autores destacan una tendencia clara hacia el aprendizaje individual, enfocado hacia el aprendizaje de vocabulario, frente al aprendizaje colaborativo y de gramática, que apenas se tratan.

\section{CONTEXTO}

El objetivo del presente trabajo es analizar las investigaciones más recientes en el ámbito de MALL a fin de ofrecer un cuadro lo más actual posible en cuanto al uso de apps y smartphones dentro de la enseñanza de idiomas. Para ello, se hará una RSL cuyo objetivo será identificar, por un lado, las principales tendencias a la hora de integrar apps en los procesos de enseñanza-aprendizaje y, por otro, comprobar las siguientes hipótesis de partida: H1: El uso de apps en la enseñanza y el aprendizaje de idiomas está en aumento y H2: El tipo de aprendizaje, que soportan la mayoría de las apps, no es muy diferente de aquel propio de la enseñanza tradicional de idiomas.

Con el objetivo de comprobar nuestras hipótesis, se llevará a cabo una RSL basada en los principios propuestos por (Kitchenham \& Charters, 2007), quienes destacan la importancia de primero justificar la necesidad de una RSL; segundo, plantear varias preguntas de investigación a fin de establecer un marco teórico para la revisión; tercero, definir una serie de términos de búsqueda y, por último, determinar y establecer diferentes bibliotecas digitales y revistas. Partiendo de la propuesta de Kitchenham y Charters, (2007) y a fin de identificar las tendencias más destacables en el ámbito de MALL, se plantean las siguientes preguntas de investigación: ¿Qué tipo de publicaciones tratan el uso de apps en los procesos de enseñanza y aprendizaje de idiomas?, ¿Qué tipo de apps se describen en ellas? y ¿Qué tipo de aprendizaje soportan las apps analizadas? Mediante el análisis de la primera pregunta se obtendrá información sobre el año y el tipo de publicaciones que se encuentran en la literatura para el período analizado (artículos en revistas, publicaciones en actas de congreso, capítulos de libro). En cambio, el análisis de la segunda pregunta permitirá establecer el tipo de alumnado y nivel lingüístico al que se dirige la mayoría de las aplicaciones móviles encontradas. Asimismo, el análisis determinará si se trata de apps específicas (especialmente diseñadas para el aprendizaje de idiomas), de apps de mensajería instantánea o de apps de alguna red social o propósito general. Finalmente, la tercera pregunta apunta a un análisis más profundo de las aplicaciones, ahondando en los tipos de aprendizaje que se encuentran (por ej. aprendizaje basado en la forma, aprendizaje por tareas o proyectos, aprendizaje basado en juegos, aprendizaje individual vs. aprendizaje colaborativo).

\section{DESCRIPCIÓN}

A fin de identificar y analizar las publicaciones más relevantes en el ámbito de interés, se han establecido para el proceso de búsqueda un total de diez bibliotecas digitales (Web of Science, IEEE Digital Library, Springer, ACM Digital Library, ScienceDirect, repositorios institucionales, The Open University, IGI-Global, Taylor \& Francis y OnlineJournals.org) y seis revistas especializadas (CALL, Language Learning \& Technology, RECALL, CALICO, JALTCALL y English Language Teaching). Asimismo, con el objetivo de completar y actualizar los datos obtenidos por otros autores (Chinnery, 2006; Viberg \& Grönlund (2012); Burston, 2013, 2014 \& 2015; Duman, 2015), el alcance de esta RSL corresponde al periodo comprendido entre 2012 y 2016.

Para delimitar la búsqueda, la cadena de búsqueda para obtener los trabajos seleccionados fue MALL AND app AND smartphone AND "language learning". No obstante, debido a que algunas bases de datos y revistas mostraban 0 resultados utilizando esta cadena de búsqueda establecida, esta fue sustituida por otras tres cadenas de búsqueda: MALL AND "language learning", app AND "language learning" y Smartphone AND "language learning”. El resultado de la búsqueda fue un total de 733 publicaciones, que son accesibles mediante Mendeley ${ }^{1}$ y FigShare ${ }^{2}$.

El siguiente paso fue descartar aquellas publicaciones que, o no cubrían el tema de investigación, o aparecían repetidas en diferentes bases de datos. Para ello, se procedió a dividir y etiquetar las publicaciones con los términos “valid paper”, “off

\footnotetext{
${ }^{1}$ https://www.mendeley.com/community/el-uso-de-apps-en-la-ensenanza-y-elaprendizaje-de-idiomas-una-revision-sistematica/documents/
} 2016/5117263 
topic” y “duplicado”. A continuación, se indican los criterios de exclusión (Tabla 1).

\section{Tabla 1. Criterios de exclusión.}

\begin{tabular}{|l|l|}
\hline \multicolumn{1}{|c|}{ Criterio } & Clasificación \\
\hline $\begin{array}{l}\text { Trabajos publicados antes del 2012 o } \\
\text { después del } 2016\end{array}$ & Off topic \\
\cline { 1 - 1 } $\begin{array}{l}\text { Trabajos que no cubren los temas de interés } \\
\text { específicos para este estudio }\end{array}$ & \\
\hline $\begin{array}{l}\text { Trabajos duplicados o escritos por los } \\
\text { mismos autores que no aportan nueva } \\
\text { información }\end{array}$ & \\
\hline
\end{tabular}

Destaca que dentro de las publicaciones etiquetadas como "off topic", había muchas que aún tratando el tema del aprendizaje con dispositivos móviles e incluso con apps, no resultaban útiles para el análisis, ya que, al ser estudios de percepción o meros resúmenes sobre el tema, no aportan los datos necesarios para este. Esto explica por qué las publicaciones a analizar en este estudio se redujeron finalmente de 733 a 57 . Una vez descartadas las publicaciones identificadas como "off topic" y "duplicados”, se empezó con el análisis exhaustivo de las 57 publicaciones válidas a fin de confirmar o desmentir las dos hipótesis iniciales de nuestro trabajo.

\section{RESUltados}

Con la idea de comprobar la $\mathrm{H} 1$ (El uso de apps en la enseñanza y el aprendizaje de idiomas está en aumento) se procedió primero a clasificar las 57 publicaciones según su año de publicación. Los resultados indican que aunque los años 2012 y 2013 estuvieran igualados, hay un pequeño aumento en el año 2014, seguido por un claro crecimiento en el año 2015 y 2016 (Figura 1).

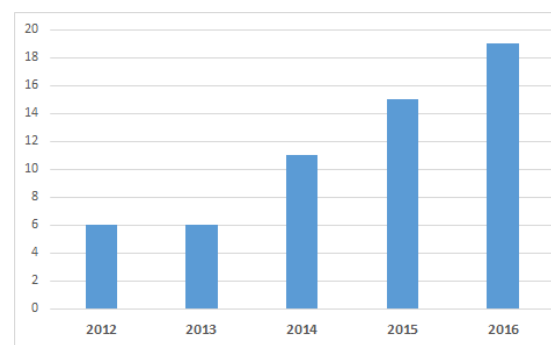

Figura 1. Número de publicaciones entre enero 2012 y diciembre

2016.

Además, los resultados de la búsqueda permitieron dar respuesta a la primera pregunta (¿Qué tipo de publicaciones tratan el uso de apps en los procesos de enseñanza $y$ aprendizaje de idiomas?). Los datos indican que hay una clara prevalencia de publicaciones en revistas científicas de impacto y actas de congreso (32 y 23 respectivamente frente a solo 2 que aparecen en libros especializados), siendo estas fuentes con más potencial de difusión y accesibilidad por parte de la comunidad científica. Otro dato interesante estuvo relacionado con el tipo de investigaciones que se llevaron a cabo. Para clasificar las publicaciones se recurrió a la propuesta de Wieringa et al. (2006), quienes distinguen entre seis tipos de trabajos de investigación: Estudios de evaluación (publicaciones que presentan la investigación de un problema o una implementación de una técnica ya existente); Estudio de validación (publicaciones que ponen en práctica una nueva propuesta que nunca había sido implementada); Propuesta de solución (publicaciones que proponen una nueva técnica, como por ejemplo una app, que incluso puede no estar totalmente desarrollada); Publicaciones filosóficas (publicaciones en las que el autor propone un nuevo marco conceptual para describir una solución); Artículos de opinión (publicaciones que proponen compartir una opinión personal del autor sobre qué se debería hacer, en vez de presentar resultados de una investigación, información sobre un diseño, etc.); Artículos de experiencia personal (publicaciones en las que el autor describe su experiencia personal). Los resultados indicaron que en las 57 publicaciones analizadas prevalen claramente los estudios de validación (27 de 57) y evaluación (17 de 57) como también los estudios con propuestas de solución (13 de 57).

Una vez comprobada la primera hipótesis (H1) se pasó al análisis de la segunda hipótesis (H2): El tipo de aprendizaje que soportan la mayoría de las apps no es muy diferente de aquel propio de la enseñanza tradicional de idiomas). Además, para obtener datos más detallados se hizo un análisis basado en las siguientes preguntas de investigación: ¿Qué tipo de apps se describen? y ¿Qué tipo de aprendizaje soportan las apps que se encuentran en las publicaciones analizadas? La primera pregunta permitió establecer el tipo de apps que predomina en las publicaciones analizadas, es decir, el número de apps que están diseñados específicamente para el aprendizaje de idiomas (41 de 57) junto al número de apps que están diseñadas como mensajería instantánea (12 de 57), red social (2 de 57) o con otro propósito (5 de 57) como apps de reconocimiento automático del habla o iTunes U.

Otro dato importante fue identificar el nivel lingüístico que trabajan las diferentes apps. Los resultados del análisis demuestran claramente que son, sobre todo, los niveles iniciales los que están más representados (23 de 57), seguidos por aquellas que están enfocados hacia los niveles intermedio (16 de 57) y avanzados (8 de 57), repartiéndose el resto entre apps que, o trabajan varios niveles, o no especifican el nivel.

La segunda pregunta pretendió hacer un análisis más detallado de las apps, analizando el contenido, enfoque y tipo de aprendizaje que estas promueven. En cuanto al contenido, se observa un claro predominio de apps que se centran en la enseñanza de vocabulario (34 de 57), seguido de lejos por otras que se centran en aspectos como la comprensión oral (15 de 57), la pronunciación (9 de 57), la gramática (8 de 57), la expresión oral (7 de 57), la expresión escrita (10 de 57), la ortografía (5 de 57), la cultura (5 de 57) etc. Los datos claramente indican que el aprendizaje de vocabulario es el que más se trabaja en las apps, mientras que aspectos como la gramática, la expresión oral o la cultura apenas se consideran. El análisis de las 57 publicaciones entre 2012 y 2016 reveló además que el enfoque de aprendizaje, que prevalece en las apps analizadas, es claramente el que promueve el aprendizaje individual (33 de 57) frente al aprendizaje colaborativo (13 de 57) o mixto (11 de 57). Otro dato interesante fue el tipo de aprendizaje que proporcionan las apps analizadas. Una vez 
más los datos indican que el tipo de aprendizaje más frecuente es el que se centra en un aprendizaje formal de la lengua, encontrando pocas apps que exploten el potencial motivador y educativo del aprendizaje gamificado o basado en juegos, del aprendizaje por tareas, etc. (Figura 2).

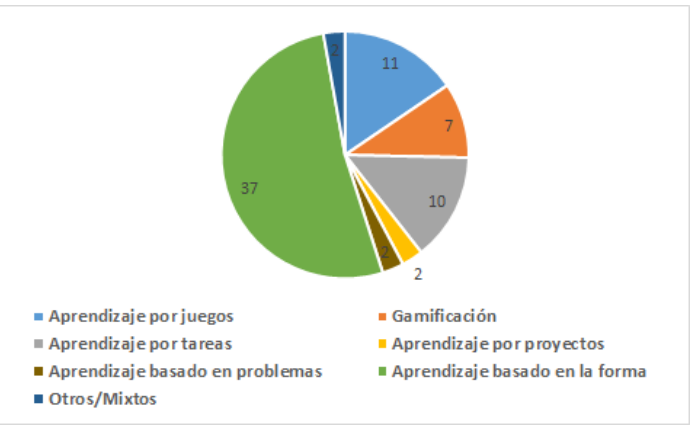

Figura 2. Tipos de aprendizaje.

Los datos demuestran, por lo tanto, que la mayoría de las apps sigue fomentando un aprendizaje centrado en aspectos formales de la lengua (37 de 57), explorando apenas las posibilidades de promover un aprendizaje interactivo y dinámico basado en la solución de problemas (2 de 57), tareas (10 de 57), etc. Asimismo, pocas apps parecen explotar el potencial motivador de elementos como la gamificación (7 de 57) o el aprendizaje mediante juegos (11 de 57).

\section{CONCLUSIONES}

El objetivo del presente estudio fue encontrar evidencias para validar las dos hipótesis iniciales de nuestro trabajo: H1: El uso de apps en la enseñanza y el aprendizaje de idiomas está en aumento y H2: El tipo de aprendizaje que soportan la mayoría de las apps no es muy diferente de aquel propio de la enseñanza tradicional de idiomas. La primera hipótesis fue confirmada por los resultados del análisis cuantitativo que se hizo. Dichos resultados demuestran claramente que el número de publicaciones en el ámbito de MALL ha ido aumentando de forma muy constante a lo largo de los últimos 5 años. La segunda hipótesis fue igualmente confirmada por los resultados del análisis cualitativo, que ha demostrado que el uso de apps en el aprendizaje y la enseñanza de idiomas no ha supuesto un cambio con respecto a los tradicionales enfoques de enseñanza-aprendizaje. Muy al contrario, el foco de atención sigue estando sobre el aprendizaje individual frente al aprendizaje colaborativo. Junto a la tendencia de fomentar el aprendizaje individual del estudiante, se observa además una clara tendencia hacia el aprendizaje centrado en aspectos formales (vocabulario, comprensión oral, pronunciación, gramática), brindando a sus usuarios pocas o ninguna posibilidad de interactuar entre ellos. Asimismo, ofrecen apenas oportunidades de trabajar destrezas como la expresión oral y escrita mediante la interacción y negociación con otros usuarios (Berns et.al., 2017). Estos resultados coinciden con los descritos por Burston (2014) años atrás, quien afirmó:

Since 2007, the emphasis in MALL has continued to be on content delivery within an implicitly behaviorist, teachercentered framework. Text-based tutorial applications involving drill and repetition of the type advocated by B.F.

Skinner (1957) continue to be the norm.

A pesar de que no todas las apps, entre las analizadas en este trabajo, comparten las mismas características, la mayoría de ellas siguen el modelo descrito anteriormente por Burston (2014). De ahí que el presente estudio confirme, una vez más, la necesidad de seguir explorando el potencial de MALL para brindar entornos y contenidos de aprendizaje, que en lugar de replicar modelos de enseñanza existentes aprovechen el potencial de las tecnologías para así enriquecer y mejorar los mismos (Stockwell y Hubbard, 2013; García-Peñalvo et al., 2017). Tales mejoras podrían consistir en crear entornos más personalizados (Humanante Ramos, et al., 2016) e interactivos, recurriendo incluso a elementos de gamificación a fin de incrementar la motivación del alumnado (Herranz et al., 2016). Asimismo, se hace necesario promover entornos y tipos de aprendizaje que son difíciles o, incluso, imposibles de recrear sin el uso de las tecnologías (p. ej. entornos que se basan en el uso de realidad aumentada o en el aprendizaje comunitario, permitiendo al propio alumnado a participar activamente en la creación y evaluación de sus materiales de aprendizaje, etc.). Algunas propuestas innovadoras en esta dirección se pueden encontrar en los estudios realizados por Berns et. al., (2015, 2016 \& 2017), Palomo-Duarte et. al. (2016), Read y Barcena (2016), Rico y Agudo (2016) y RuizRube et.al. (2016).

\section{REFERENCIAS}

Berns, A., Palomo-Duarte, M., Dodero, J. M., Ruiz-Ladrón, J. M., y Calderón Márquez, A. (2015). Mobile apps to support and assess foreign language learning. En F. Helm, L. Bradley, M. Guarda, y S. Thouësny (eds.), Critical CALL - Proceedings of the 2015 EUROCALL Conference. Conferencia llevada a cabo en Padova.

Berns, A., Isla-Montes, J.L., Palomo-Duarte, M., y Dodero, J.M. (2016). Motivation, students' needs and learning outcomes: a hybrid game-based app for enhanced language learning. SpringerPlus 5 (1), p. 1305.

Berns, A., Palomo-Duarte, M., Isla-Montes, J.L., Dodero, J.M. y Delatorre, P. (2017). Agenda colaborativa para el aprendizaje de idiomas: del papel al dispositivo móvil. Ried 20 (2), p. 119-139.

Briz-Ponce, L., Juanes-Méndez, J. A., y García-Peñalvo, F. J. (2016). Handbook of Research on Mobile Devices and Applications in Higher Education Settings. Hershey, PA: IGI Global.

Burston, J. (2013). Mobile-Assisted Language Learning: a Selected Annotated Bibliography of Implementation Studies. Language Learning \& Technology, 17 (3), pp.157-225.

Burston, J. (2014). MALL: the pedagogical challenges. Computer Assisted Language Learning, Routledge, Taylor \& Francis Group, 27 (4), pp. 344-357.

Burston, J. (2015). Twenty years of MALL project implementation: A meta-analysis of learning outcomes. ReCALL, 27 (1), pp. 4-20. 
Chinnery, G.M. (2006). Going to the MALL: Mobile Assisted Language Learning. Language Learning \& Technology, 10, (1), pp. 9-16.

Duman, G., Orhon, G. y Gedik, N. (2015). Research trends in mobile assisted language learning from 2000 to 2012, ReCALL, 27 (2), pp. 197-216.

García-Peñalvo, F. J., Hernández-García, A., Conde, M. A., Fidalgo-Blanco, A., Sein-Echaluce, M. L., AlierForment, M., Llorens-Largo, F. y Iglesias-Pradas, S. (2017). Enhancing Education for the Knowledge Society Era with Learning Ecosystems. En F. J. GarcíaPeñalvo y A. García-Holgado. (Ed.), Open Source Solutions for Knowledge Management and Technological Ecosystems (pp. 1-24). Hershey, PA: IGI Global.

Heil, C. R., Wu, J. S., Lee, J. L., y Schmidt, T. (2016). A review of mobile language learning applications: trends, challenges and applications. En The EUROCALL Review, 24 (2), pp. 32-50.

Herranz, E., Colomo-Palacios, R., de Amescua Seco, A. y Sánchez-Gordón, M. (2016). Towards a Gamification Framework for Software Process Improvement Initiatives: Construction and Validation. Journal of Universal Computer Science, 22, (12), pp. 1509-1532.

Humanante Ramos, P., García-Peñalvo, F. J., y CondeGonzález, M. (2017). Entornos personales de aprendizaje móvil: una revisión sistemática de la literatura. RIED. Revista Iberoamericana de Educación a Distancia, 20, (2), pp. 73-92

Kitchenham, B. y Charters, S. (2007). Guidelines for performing Systematic Literature Reviews in Software Engineering, Elsevier, 2, p. 1051.

Kukulska-Hulme, A. (2005). Introduction. En A. KukulskaHulme \& J. Traxler, Routledge (eds.), Mobile Learning: a handbook for educators and trainers, pp. 1-7. Great Britain.

Kukulska-Hulme, A., y Shield, L. (2008). An overview of mobile assisted language learning: From content delivery to supported collaboration and interaction, ReCALL, 20, (3), pp. 271-289.

Palomo-Duarte, M., Berns, A., Cejas, A., Dodero, J.M. y Caballero, J.A. (2016). Assessing foreign language learning through mobile game-based learning environments, International Journal of Human Capital and Information Technology Professionals, IGI Publishing Hershey, 7, (2), pp. 53-67.

Pinto-Llorente, A. M., Sánchez-Gómez, M. C., y GarcíaPeñalvo, F.J. (2016). Assessing the Effectiveness of Interactive and Collaborative Resources to Improve Reading and Writing in English, International Journal of Human Capital and Information Technology Professionals, IGI Publishing, 7, (1), pp. 66-85.

Ramírez-Montoya, M. S. y García-Peñalvo, F. J. (2017). La integración efectiva del dispositivo móvil en la educación y en el aprendizaje. RIED. Revista Iberoamericana de Educación a Distancia, 20, (2), pp. 29-47.

Read, T., y Barcena, E. (2016). Metacognition as scaffolding for the development of listening comprehension in a social MALL App. RIED. Revista Iberoamericana de Educación a Distancia, 19, (1), pp. 103-120.

Rico, M. M., y Agudo, J. E. (2016). Aprendizaje móvil de inglés mediante juegos de espías en Educación Secundaria. RIED. Revista Iberoamericana de Educación a Distancia, 19, (1), pp. 121-139.

Ruiz-Rube, I., Mota, J. M., Person, T., Berns, A., y Dodero, J. M. (2016). Autoría y analítica de aplicaciones móviles educativas multimodales. En XVIII Simposio Internacional de Informática Educativa SIIE 2016.

Stockwell, G., y Hubbard, P. (2013). Some Emerging Principles for Mobile-assisted Language Learning. The International Research Foundation for English Language Education. Recuperado de http://www.tirfonline.org/english-in-theworkforce/mobile-assisted-language-learning/someemerging-principles-for-mobile-assisted-languagelearning/

Viberg, O., y Grönlund, A. (2012). Mobile Assisted Language Learning: A Literature Review. mLearn, Conference on Mobile and Contextual Learning. Recuperado de https://pdfs.semanticscholar.org/e1e7/b91e3c470766231 04ff8ec296902497141b9.pdf?_ga=2.61254700.1206417 57.1500030324-1684578701.1497085438

Wieringa, R., Maiden, N. A. M., Mead, N. R. y Rolland, C. (2006). Requirements engineering paper classification and evaluation criteria: a proposal and a discussion, Requir. Eng., Springer-Verlag, 11, (1), pp. 102-107. 